2

\title{
Optogenetic manipulation of YAP cellular localisation and function
}

\author{
P. J. Y. Toh ${ }^{1}$, J. K. H. Lai ${ }^{1}$, A. Hermann ${ }^{2}$, O. Destaing ${ }^{3,4,5}$, \\ M. P. Sheetz ${ }^{1,6^{*}}$, M. Sudol ${ }^{1,7^{*}}$ and T. E. Saunders ${ }^{1,8,9^{*}}$ \\ ${ }^{1}$ Mechanobiology Institute, National University of Singapore, Singapore \\ ${ }^{2}$ University of Münster, Germany \\ ${ }^{3}$ Université Grenoble Alpes, Institute for Advanced Biosciences, Grenoble 38042, France \\ ${ }^{4}$ INSERM U1209, Institute for Advanced Biosciences, F-38700 La Tronche, France \\ ${ }^{5}$ CNRS UMR 5039, Institute for Advanced Biosciences, F-38700 La Tronche, France \\ ${ }^{6}$ Department of Biochemistry and Molecular Biology, University of Texas Medical Branch, Galveston, USA \\ ${ }^{7}$ Icahn School of Medicine at Mount Sinai, New York City, USA \\ ${ }^{8}$ Institute of Molecular and Cell Biology, A*STAR, Singapore \\ ${ }^{9}$ Warwick Medical School, University of Warwick, United Kingdom \\ * For correspondence: M.P.S. (misheetz@utmb.edu), M.S. (marius.sudol@mssm.edu), \\ T.E.S. (timothy.saunders@,warwick.ac.uk)
}

\section{$\underline{\text { Abstract }}$}

YAP, an effector of the Hippo signalling pathway, promotes organ growth and regeneration. Prolonged YAP activation results in uncontrolled proliferation and cancer. Therefore, exogenous regulation of YAP activity has potential translational applications. We present a versatile optogenetic construct (optoYAP) for manipulating YAP localisation, and consequently its activity and function. We attached a LOV2 domain that photocages a nuclear localisation signal (NLS) to the N-terminus of YAP. In $488 \mathrm{~nm}$ light, the LOV2 domain unfolds, exposing the NLS, which shuttles optoYAP into the nucleus. Nuclear import of optoYAP is reversible and tuneable by light intensity. In cell culture, activated optoYAP promotes YAP target gene expression, cell proliferation, and anchorage-independent growth. Similarly, we can utilise optoYAP in zebrafish embryos to modulate target genes. OptoYAP is functional in both cell culture and in vivo, providing a powerful tool to address basic research questions and therapeutic applications in regeneration and disease.

Keywords: YAP, Optogenetics, Cell proliferation, Spatiotemporal dynamics, Zebrafish 


\section{Introduction}

The Hippo signalling pathway regulates organ size control and cell fate during development and regeneration. ${ }^{1}$ Components of this pathway were identified in a Drosophila melanogaster genetic screen ${ }^{2,3}$ and are evolutionary conserved. ${ }^{4,5}$ The core kinases in the Hippo pathway are MST1/2 and LATS1/2. MST1/2 phosphorylates and activates LATS1/2 ${ }^{6}$ in the presence of $\mathrm{SAV}^{7}$ and MOB1A/B. ${ }^{8}$ Subsequently, activated LATS1/2 phosphorylates YAP/TAZ ${ }^{3}$ which results in their cytoplasmic sequestration from the nucleus by 14-3-3 proteins ${ }^{9,10}$ or their degradation. ${ }^{11}$

Cellular responses to Hippo signalling include proliferation, migration, changes in cellular cytoskeleton and morphology, as well as cell survival. ${ }^{12}$ Genetic loss of the Hippo kinases or hyperactivation of YAP/TAZ (Yorkie in D. melanogaster), has been shown to promote tissue regeneration, ${ }^{13-15}$ but at the risk of organ overgrowth. ${ }^{4,16,17}$ Furthermore, YAP/TAZ are also key drivers in tumour development. ${ }^{18}$ Therefore, to harness the therapeutic benefits of YAP/TAZ without suffering from their tumorigenic potential, developing a tool to control the activation of YAP is desirable.

Optogenetics is a powerful method for controlling the spatiotemporal localisation and activation of specific biological processes. It has been used extensively to activate neural responses. ${ }^{19}$ Optogenetic strategies have also been deployed to modulate and probe cell signalling pathways ${ }^{20}$ where high spatiotemporal control is desirable, such as Ras/Erk, ${ }^{21} \mathrm{Akt},{ }^{22}$ Notch, ${ }^{23}$ Bicoid, ${ }^{24} \mathrm{Src}^{25}$ and p53 signalling pathways. ${ }^{26}$ In light of the variety of optogenetic tools and capabilities, optogenetic approaches potentially enable precise regulation of the YAP/TAZ nuclear-cytoplasmic localisation, which can modulate their co-transcriptional activity.

To this end, we utilised the LOV2-J $\alpha$ interacting domain to photocage a NLS within the J $\alpha$ helix domain. ${ }^{27}$ Fusing this light inducible domain to the N-terminus of YAP, henceforth referred to as optoYAP, thus enables manipulation of YAP cellular localisation with light, which we report here. We show that optoYAP is imported into the nucleus after a $488 \mathrm{~nm}$ light activation protocol in a range of cell lines and in the zebrafish embryo. We find that the optoYAP nuclear import dynamics are tuneable by activation light intensity. Activated optoYAP elicits cellular responses including the expression of downstream target genes and cell proliferation. Strikingly, we find that optoYAP can promote anchorage-independent 
67

growth, showing that optoYAP activation is sufficient to override the mechano-inhibitory signals in cells seeded on soft growing substrates. Such specific control opens new opportunities to explore key questions concerning dysregulation of YAP localisation and its effects on disease progression. Further, it enables the potential for the manipulation of YAP/TAZ activity in therapeutic applications.

\section{$\underline{\text { Results }}$}

Optogenetic YAP construct: To tightly control YAP localisation both temporally and spatially, we fused an optogenetic construct to the N-terminal end of hYAP1. ${ }^{27}$ This construct consists of the LOV2 domain of Avena sativa phototropin 1 with mCherry for visualisation (Fig. 1A). The LOV2 domain is connected to hYAP1 via a J $\alpha$ helix and NLS sequence. A nuclear export signal (NES) derived from truncated cAMP-dependent protein kinase (PKI) facilitates nuclear export and decreases background nuclear localisation in the dark state. ${ }^{27}$ We refer to this construct as optoYAP (Fig. 1A).

In the dark, the LOV2 domain photocages the $\operatorname{NLS}^{27}$ to prevent importation of optoYAP into the nucleus (Fig. 1A). Under blue light illumination, the J $\alpha$ helix domain unfolds and is released from the LOV2 domain to expose the NLS and allows transport of optoYAP into the nucleus.

Characterisation of optogenetic YAP in mammalian cells: HEK293T cells were transiently transfected with optoYAP and subjected to $488 \mathrm{~nm}$ pulsatile illumination of $1 \mathrm{~s}$ every $30 \mathrm{~s}$, similar to optogenetic activation protocols described previously ${ }^{27}$ (Fig. 1A). Localisation of optoYAP is then visualised by the attached mCherry fluorophore. Whereas optoYAP is localised to the cytoplasm without activation (Fig. 1B-B'), its accumulation in the nucleus doubles after 20 minutes of activation (Fig. 1C-C' and Supplementary Video 1).

We tested whether optoYAP shuttles in and out of the nucleus by recurring activation cycles. We subjected HEK293T cells to three rounds of activation protocol (20 minutes) and recovery in the dark (20 minutes). OptoYAP accumulates in the nucleus to the same level when activated and retreats to its baseline level after each activation cycle (Fig. 1D and Fig. S1A). This property shows that optoYAP activation is reversible and its light-sensitive property is not attenuated over activation cycles. 
101 To explore the nuclear import and export dynamics of optoYAP, we performed time-lapse

102

103

104

105

106

107

108

109

110

111

112

113

114

115

116

117

118

119

120

121

122

123

124

125

126

127

128

129

130

131

132 imaging of optoYAP in HEK293T cells. Cells were exposed to the above activation protocol for 20 minutes and then the cells were left in the dark for a further 20 minutes, while optoYAP localisation was tracked every 30 seconds. The nuclear accumulation saturated quickly, with an activation time constant $\left(\tau_{\mathrm{a}}\right)$ of $5.6 \pm 0.4$ minutes (Fig. 1E, Fig. S1B and Methods). The recovery time constant $\left(\tau_{\mathrm{r}}\right)$ was $28.1 \pm 2.4$ minutes (Fig. 1E and Fig. S1C).

We next tested the light sensitivity of optoYAP in HEK293T cells. We performed a series of light activations using varying $488 \mathrm{~nm}$ laser power, $0.065,0.162$, and $0.325 \mathrm{~mW}$, measured at the focal plane. Both $\tau_{\mathrm{a}}$ (Fig. S1D) and extent of nuclear accumulation of optoYAP positively responded to increasing laser powers (Fig. 1F).

Phosphorylation of YAP at serine 127 residue has been shown to promote its cytoplasmic localisation. ${ }^{28} \mathrm{We}$ investigated the phosphorylation status of optoYAP when it is nuclear localised after light activation. Using CRISPR-mediated YAP knockout MKN28 cells, we transfected optoYAP into these cells and probed for pYAP (S127) using Western blot (Fig. S1E). Phosphorylated optoYAP was clearly present in the nucleus, suggesting that the NLS in our optoYAP construct can import phosphorylated optoYAP marked for cytoplasmic sequestration.

Functionality of nuclear-localised optoYAP: We next investigated the functionality of optoYAP. As YAP is a transcriptional co-regulator, it binds to TEAD transcription factors in the nucleus to initiate transcription of downstream genes. ${ }^{29}$ HEK293T cells transiently transfected with optoYAP were subjected to pulsed light activation over $48 \mathrm{~h}$ followed by qPCR assays of YAP target genes: ANKRD1, CTGF and CYR61. The expression levels of all three genes were significantly upregulated in cells with activated optoYAP construct as compared to control cells without light activation (Fig. 2).

Given that activated optoYAP can activate downstream targets, we postulated that activated optoYAP could also promote cells to proliferate. We assayed cell proliferation of two cell lines, HEK293T and HFF, by using a DNA-binding fluorescent dye to measure their total DNA. Cells were transfected with optoYAP and subjected to pulsatile light activation over one week. 
133

134

135

136

137

138

139

140

141

142

143

144

145

146

147

148

149

150

151

152

153

154

155

156

157

158

159

160

161

162

163

164

165

166

Both transformed (HEK293T, Fig. 3A) and non-transformed (HFF, Fig. 3B) cell lines show significant increase in total DNA with activated optoYAP as compared to untransfected and unactivated controls.

optoYAP can induce anchorage independent growth: Mechanical parameters of the microenvironment, such as matrix rigidity, are crucial for normal cell growth. ${ }^{30}$ In particular, epithelial cells typically cannot survive and proliferate on soft substrates. However, transformed cells are able to bypass rigidity sensing and grow on soft substrates. ${ }^{31} \mathrm{We}$ hypothesised that activated optoYAP may drive proliferation of cells even on soft substrates. Using a non-transformed cell line, HFF, we cultured untransfected and optoYAP-transfected cells with or without light activation on soft agar for seven days. As expected, few untransfected cells survived, with little visible colony formation (Fig. 3C). However, optoYAP-transfected cells that were subjected to light activation formed more colonies (Fig. 3C and Fig. S2A) with larger size (Fig. 3C') compared to those without light activation. The increase in number and size of colonies formed indicates that activated optoYAP can induce transformed growth, overriding the ability of normal cells to sense their microenvironment and suppress growth in soft substrates.

Similarly, we performed the soft agar assay on a transformed cell line MKN28. Using $Y A P^{-/-}$ MKN28 cells, ${ }^{32}$ we rescued these mutant cells with either eGFP-YAP or optoYAP. We seeded four variants of MKN28 cells - wildtype (WT), $Y A P^{-/}$, $Y A P^{-/-}+$eGFP-YAP, and $Y A P^{-/}+$optoYAP - on soft agar for seven days. WT MKN28 cells, unlike HFF cells, are transformed cells and are able to form colonies on soft agar, which is attenuated by the loss of YAP (Fig. 3D-3D' and Fig. S2B). While overexpression of eGFPYAP rescues YAP null MKN28 cells to form colonies at WT levels, activated optoYAP exaggerates colony formation of MKN28 cells on soft agar (Fig. 3D and Fig. S2B). Protein expression levels of both eGFP-YAP and optoYAP transfected in YAP null cells were similar (Fig. S2C). However, optoYAP was localised in the nucleus during the activation protocol, therefore likely inducing cell proliferation and increasing the number of colonies formed (Fig. 3D' and Fig. S2B). The colony size did not vary between the different MKN28 cell lines, with the exception of YAP null cells, but the number of colonies formed with activated optoYAP is more than double of the WT or eGFP-YAP rescue (Fig. S2B). These data show that exogenous and sustained activation of optoYAP in MKN28 cells triggers greater levels of cell growth on soft agar than that of WT or rescued YAP mutant cells. 
optoYAP in zebrafish embryos: Building on the functional results in mammalian cell culture, optofYap mRNA were imaged at the animal pole (Fig. 4A). optofYap is distributed uniformly in the entire cell (though we observed mosaic levels of expression between cells), prior to activation (Fig. 4B). Using a $488 \mathrm{~nm}$ pulsatile light activation protocol as per Fig. 1A, we see that optofYap can shuttle into the nuclei of both the enveloping layer (EVL) and deep cells (Fig. 4B and Supplementary Video 2). We observe a two-fold change in nuclear localisation after light activation (Fig. 4B'). Interestingly, optofYap appears to enter and leave the nucleus much faster in the zebrafish embryos than optoYAP does in cultured cells. The activation time constant was calculated to be $2.9 \pm 0.8$ minutes and recovery time constant at $4.8 \pm 1.6$ minutes (Fig. 4C and Fig. S3A-B).

Similar to our tissue culture studies, we tested if optofYap can induce the expression of specific Yap target genes in the embryo after activation. ${ }^{33}$ qPCR was performed on embryos subjected to overnight activation protocol for 14 hours from the tailbud stage (10 hours post fertilisation (hpf)). Expression of arhgap27 did not change in all conditions, while ctgfa, vgll2b and vgll4b were upregulated after activation. Even though cyr61 was upregulated by optofYap, contribution by the activation protocol is unclear (Fig. 4D).

\section{$\underline{\text { Discussion }}$}

189 We report the development of a functional optogenetic YAP, optoYAP, by exploiting the lightresponsive LOV2-J $\alpha$ domain to photocage NLS. We characterised optoYAP function in human cells and zebrafish embryos, showing a consistent increase in YAP nuclear localisation upon blue light illumination within minutes. This rapid manipulation of YAP localisation elicits downstream responses, including the upregulation of target genes, increased cell proliferation in cell culture, and anchorage-independent growth. Long term activation of optoYAP does not appear to have detrimental effects in cell culture. Each activation cycle is independent (Fig. 1D), and cells survive after being subjected to one week of light activation. This adds to the advantage of optogenetic YAP over constitutively active YAP or drug-induced YAP expression. 
200

201

202

203

204

205

206

207

208

209

210

211

212

213

214

215

216

217

218

219

220

221

222

223

224

225

226

227

228

229

230

231

232

A similar optogenetic YAP based on the LOV2-TRAP tool has recently been described. ${ }^{34}$ However, the construct does not show a functional YAP response upon activation. There, LOV2 is tethered on the outer surface of the mitochondria and YAP is tagged with a small peptide Zdk which interacts with the LOV2 domain. ${ }^{34}$ This system requires two-plasmids to be introduced into the tissue of interest. Our optogenetic YAP construct described here requires only a single component, the LOV2-J $\alpha$ interacting domain, thus simplifying its deployment and presents an advantage for future applications. Activation of the photosensitive domain can be achieved within visible light range at $488 \mathrm{~nm}$ and recovery done in the dark. These features add to the ease of use over other optogenetic approaches that require multiple components or activation in the infrared spectrum. ${ }^{35}$ Further, to the best of our knowledge, this is the first report of a functional optogenetic YAP construct.

We observed two differences in our optogenetic construct between tissue culture cells and zebrafish embryos. Firstly, without activation, optofYap is uniformly distributed in zebrafish cells (Fig. 4B), in contrast to optoYAP which is localized to the cytoplasm of human cell lines (Fig. 1C). Secondly, the activation time constant is faster in zebrafish (2.9 min, Fig. 4C) than in cultured cells (5.6 min, Fig. 1E). The NLS and NES present in this optogenetic construct were optimized from human c-Myc and PKI, respectively. ${ }^{27}$ These two peptide sequences differ from their respective Danio rerio orthologue (Fig. S3C-D). This sequence divergence suggests that the nuclear import/export machineries in the zebrafish may be more sensitive to modified c-Myc NLS and could explain the differing dynamics of optoYAP between human and zebrafish cells. The nuclear import and export rates also differ in cell culture (Fig. 1E), which suggests that here the rate of nuclear import driven by the unmasked NLS is greater than the rate of nuclear export driven by the NES.

We found that a transformed cell line, MKN28, depends on YAP for anchorage-independent growth, as the removal of $Y A P$ results in fewer and smaller colonies in the soft agar assay. Moreover, forced overexpression of eGFP-YAP in these mutant cells reinstated colony growth to similar to WT cell levels. On the other hand, light activation of optoYAP further amplified cell proliferation by increasing the number of colonies formed. One possibility is that eGFPYAP is limited by upstream signals including Hippo kinases, and that our optoYAP appears to override such limitations as seen in our Western blot data (Fig. S1E). This shows that optoYAP might be able to bypass canonical Hippo signalling regulation. 
234 There is a potential for induced activation of YAP to promote regeneration of injured organs. ${ }^{36}$

235 However, sustained activation can lead to uncontrolled proliferation. ${ }^{37}$ YAP has been shown

236 to be required for liver regeneration after partial hepatectomy, where regenerating hepatocytes

237 had increased YAP activity that returned to original levels once liver size was restored. ${ }^{38}$ On

238 the other hand, in an organ with low regenerative capacity like the heart, overexpression of

239 YAP is sufficient to promote myocardial proliferation and cardiac regeneration. ${ }^{15,39}$ Although

240 constitutively-active YAP in cardiomyocytes increases ventricular wall thickness and ejection

241 fraction, its chronic activity is fatal. ${ }^{40}$ Therefore, heart regeneration represents an opportunity

242 for the application of optoYAP to limit the activity of YAP for cardiomyocyte renewal.

244 Overall, given the importance of YAP with respect to development and regeneration, and the 245 ease of the optogenetic tool presented here, optoYAP has the potential to become a powerful 246 tool for studying Hippo-YAP signalling in both research and clinical applications.

\section{Acknowledgements}

249 This work was supported by a Singapore Ministry of Education Tier 3 grant (MOE2016-T3-1250 002). We thank Richard De Mets for his help on data analysis. We thank members of the 251 Saunders, Sudol, and Sheetz labs for feedback on the project.

\section{Competing interests}

254 The authors declare no competing interests.

\section{$\underline{\text { Author Contributions }}$}

257 P.J.Y.T., M.S. and M.P.S. conceived the study. P.J.Y.T., J.K.H.L., M.S., M.P.S., T.E.S. 258 designed and planned the experiments. P.J.Y.T. performed the cell culture and zebrafish experiments. J.K.H.L. assisted with the experiments, particularly in the zebrafish work. A.H. generated the initial optoYAP clone with the help of O.D.. P.J.Y.T. analyzed the data with assistance from J.K.H.L. and T.E.S.. P.J.Y.T., J.K.H.L. and T.E.S. wrote the first draft of the manuscript with all authors approving the final manuscript. 


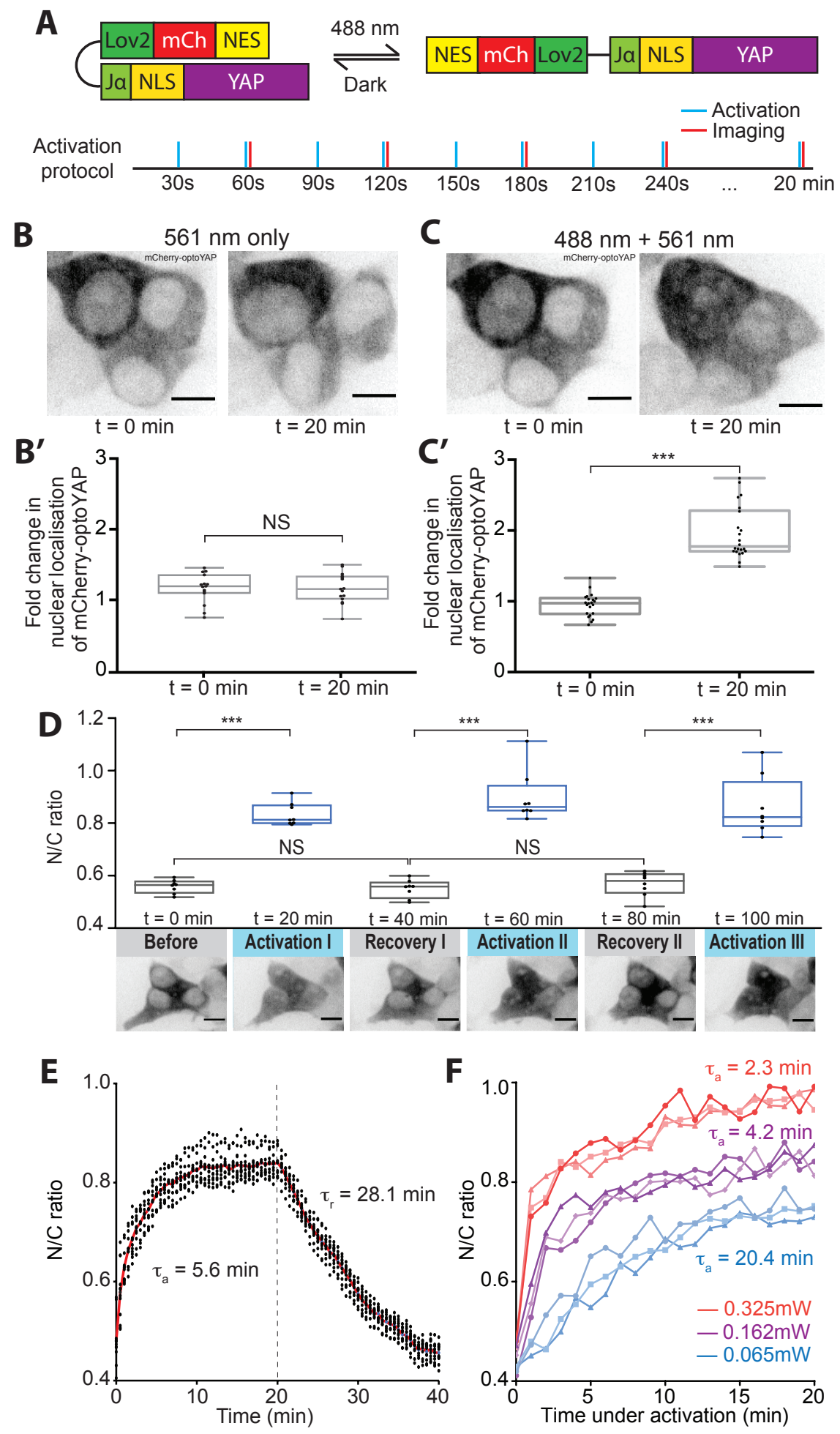

263 Figure 1. Characterisation of optoYAP in cell culture. (A) Schematic of optoYAP construct.

264 OptoYAP is folded in the dark due to the interaction between LOV2 domain and J $\alpha$ helix.

265 Under $488 \mathrm{~nm}$ light, nuclear localisation signal (NLS) is exposed and optoYAP is transported

266 into the nucleus. Activation protocol was performed with $1 \mathrm{~s}$ blue light pulse every $30 \mathrm{~s}$ (blue

267 bars), and cells were imaged every minute (red bars) for 20 minutes. (B) Representative images

268 of mCherry-optoYAP in HEK293T cells imaged with only $561 \mathrm{~nm}$ laser following the protocol 
in (A). (B') Fold-change in nuclear localisation of mCherry-optoYAP ( $\mathrm{n}=22$ cells from 2 independent experiments). (C) Representative images of the same cells in (B) exposed to both

271488 and $561 \mathrm{~nm}$ following the protocol shown in (A). (C') Fold-change in nuclear localisation 272 of the mCherry-optoYAP ( $\mathrm{n}=22$ cells from 2 independent experiments). (D) HEK293T cells 273 transfected with optoYAP were subjected to three cycles of activation protocol and recovery 274 in the dark. (Top) The ratio of mCherry-optoYAP signal in the nucleus to the cytoplasmic 275 mCherry-optoYAP signal ( $\mathrm{n}=8$ cells from 2 independent experiments). (Bottom) 276 Representative images of the mCherry-optoYAP signal from the same HEK293T cells at each 277 cycle. (E) Activation time constant $\left(\tau_{\mathrm{a}}\right)$ and recovery time constant $\left(\tau_{\mathrm{r}}\right)$ (see Methods and Fig. 278 S1B-C) of optoYAP in HEK293T cells. Vertical dashed line represents time when $488 \mathrm{~nm}$ 279 stimulation ceased. Red line indicates average nuclear/cytoplasmic ratio ( $\mathrm{n}=12$ cells). (F) 280 HEK293T cells transfected with optoYAP subjected to $0.065,0.163$ and $0.325 \mathrm{~mW} 488 \mathrm{~nm}$ laser power at the focal plane. $\mathrm{n}=3$ cells per laser power. NS: Not significant, $* * * P<10^{-3}$. Box plots represent median and $25^{\text {th }}$ to $75^{\text {th }}$ percentiles. Bars show minimum and maximum points.
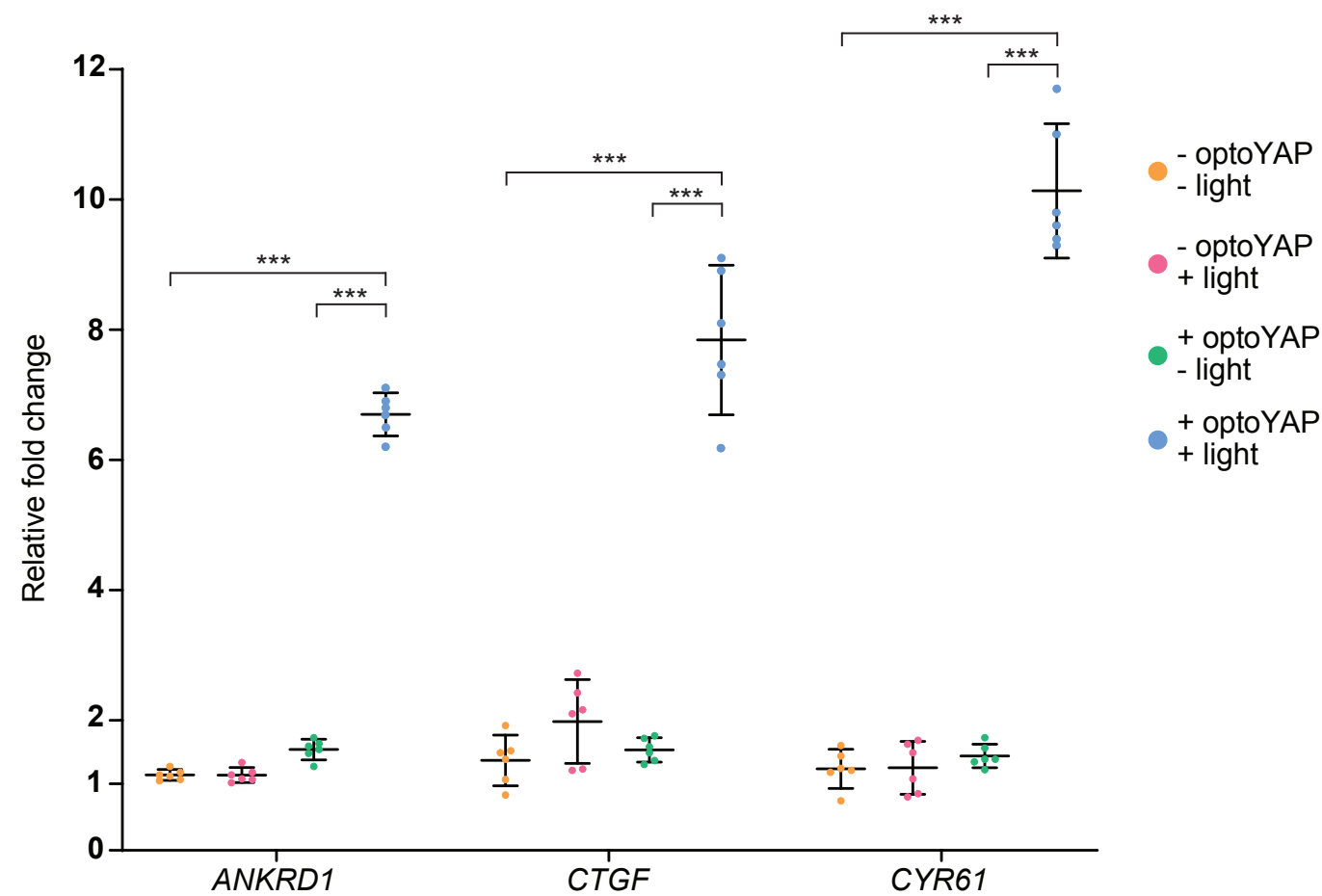

+ optoYAP

- light

+ optoYA
+ light

$A N K R D 1$

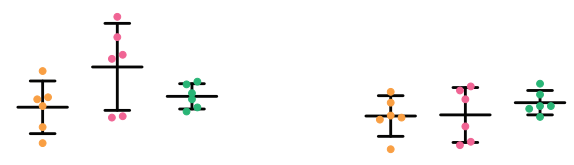

CYR61

284 Figure 2. qPCR data of downstream YAP target genes. Expression levels of $A N K R D 1$, $C T G F$, and CYR61 transcripts in HEK293T cells transfected with optoYAP after $48 \mathrm{~h}$ of activation protocol. Gene expression level was normalized to the housekeeping gene, EIF1B. Horizontal bars represent mean and 95\% confidence interval from 6 biological replicates across 2 independent experiments for each condition, $* * * P<10^{-3}$. 

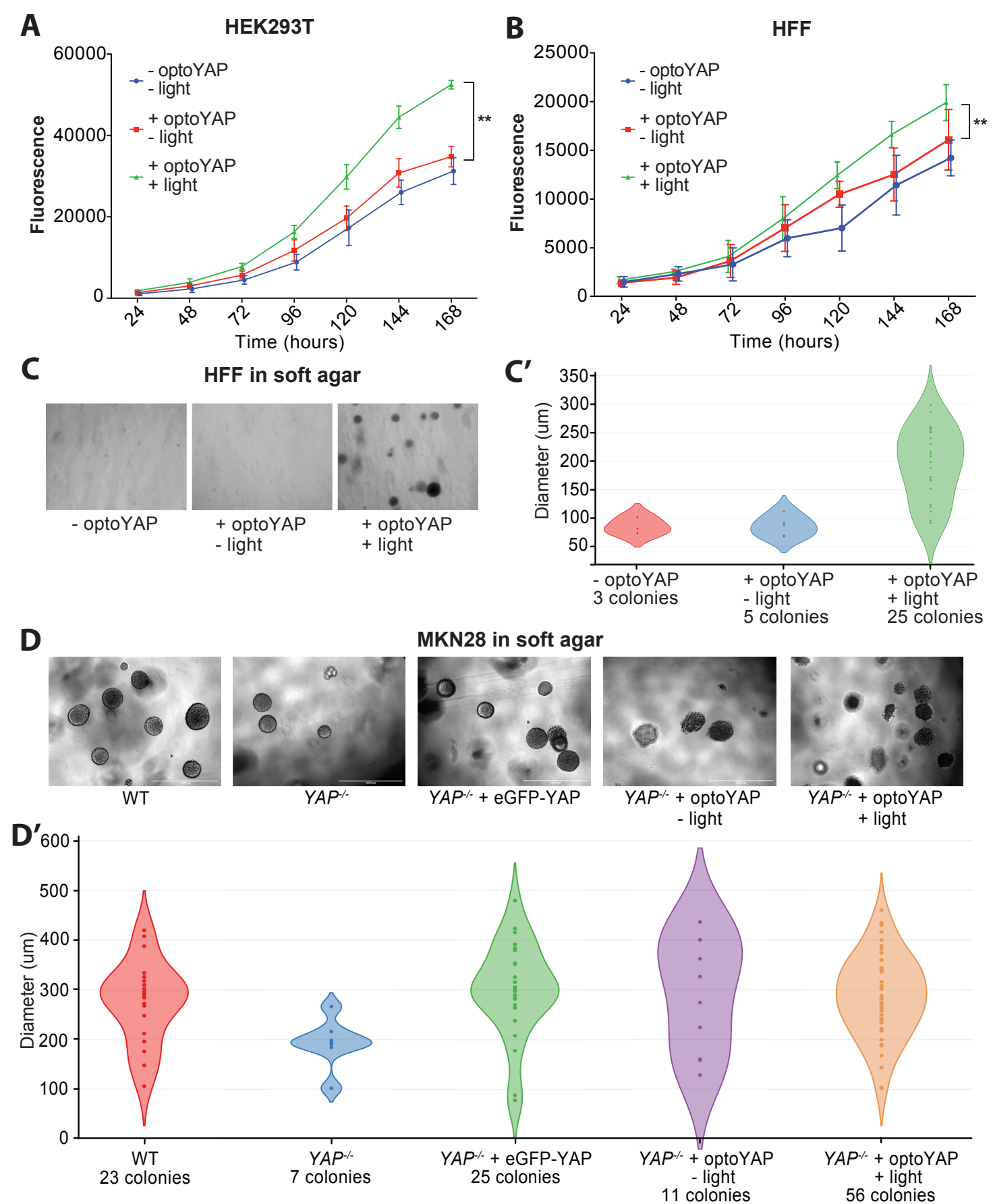

Figure 3. Functional assays of optoYAP in tissue culture cells. (A-B) Cell proliferation assay. HEK293T (A) and HFF (B)

(B) cells were transfected with optoYAP and subjected to activation protocol as described in Fig. 1A or kept in the dark for one week. Fluorescence measures amount of DNA present in each well (Methods). Error bars are s.d., $n=3$ independent experiments for each condition, ${ }^{* *} P<10^{-2}$. (C-D) Soft agar colony formation assay. HFF (C) and MKN28 (D) cells grown on soft agar for one week under different light conditions as described in Fig. 1A. (C'-D') Diameter of individual colonies formed under different light conditions and genetic backgrounds. Colonies were counted and measured from 3 biological replicates. 
A

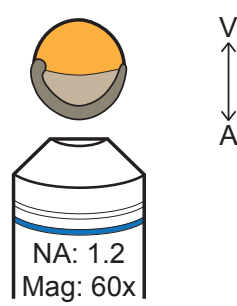

B

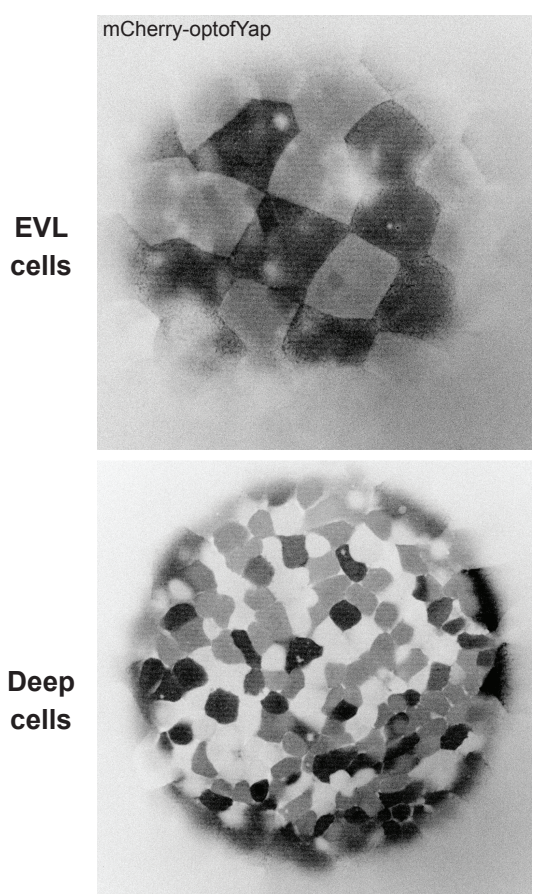

C

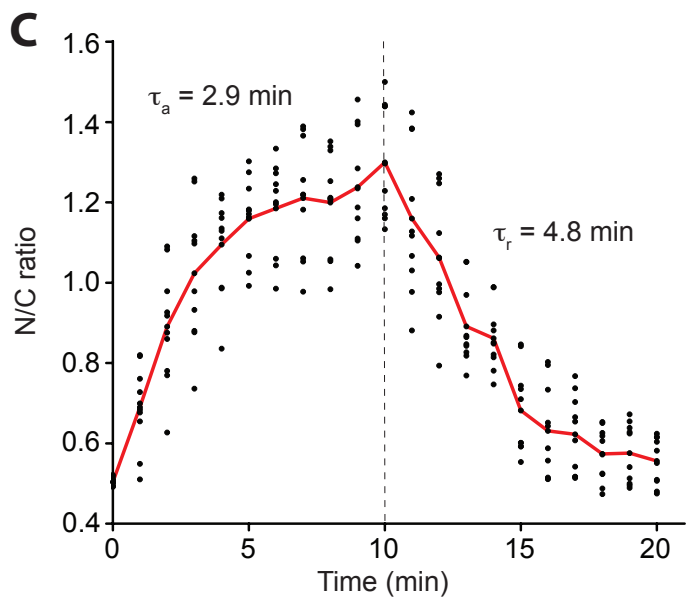

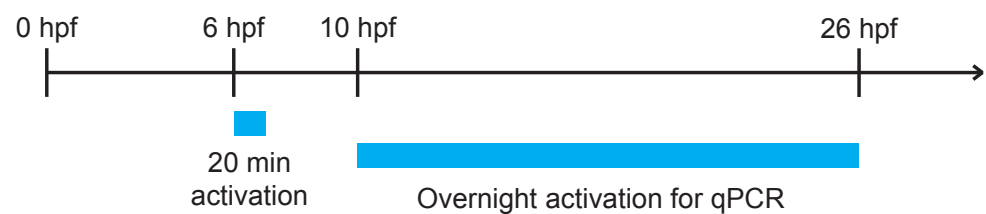

Light

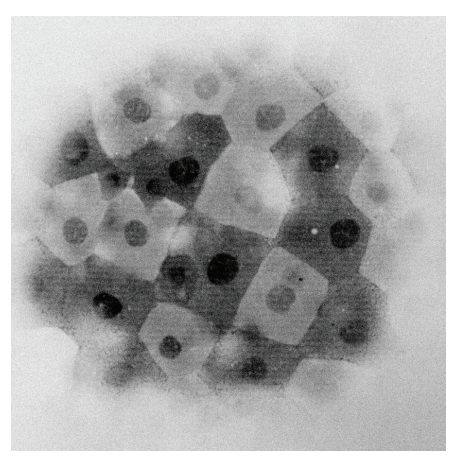

$\mathbf{B}^{\prime}$
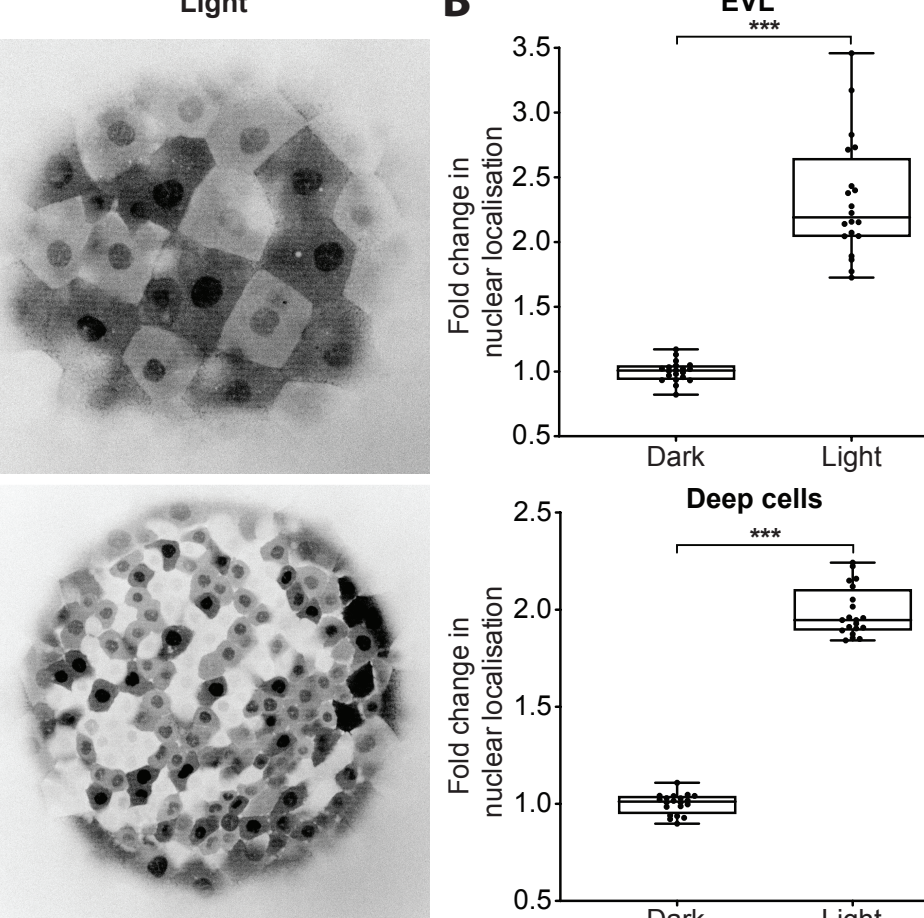

D

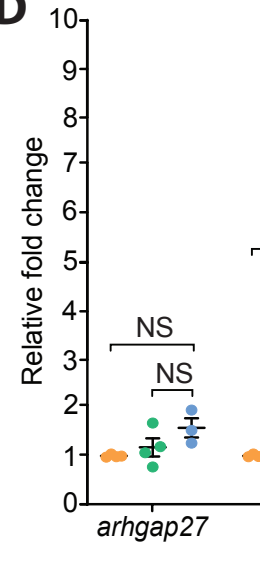

$\stackrel{* * *}{\longrightarrow}$

- light

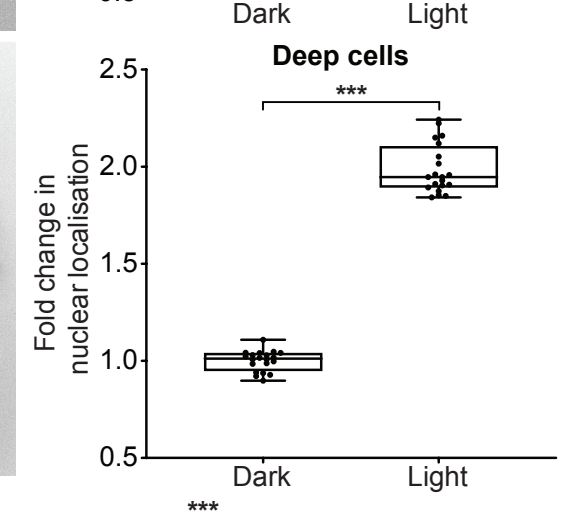

- optofYap

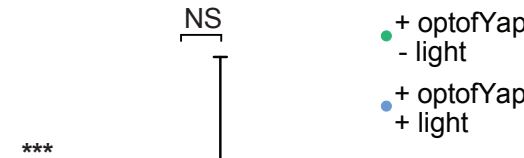





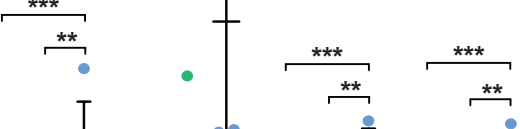

Figure 4. Validation of optofYap in zebrafish. (A) Imaging and light activation protocol in zebrafish. Shield-stage embryos were subjected to activation protocol as described in Fig. 1A and imaged at the animal pole. (B) Example images of embryos expressing optofYap (mCherry-tagged) in the EVL and deep cells kept in the dark or subjected to the activation protocol at 6 hpf. (B') Fold-change in nuclear localisation of mCherry-optofYap ( $\mathrm{n}=20$ cells 
305

306

307

308

309

310

311

312

Whiskers show minimum and maximum points. (C) The N/C ratio measured using mCherry signal in zebrafish embryos injected with optofYap tracked over 10 min of activation protocol and 10 min recovery in the dark (see also Fig. S3A-B). ( $n=10$ cells from 2 independent experiments, red line $=$ average). Vertical dashed line represents time when $488 \mathrm{~nm}$ stimulation ceased. (D) qPCR of Yap target genes. Embryos were kept in the dark until $10 \mathrm{hpf}$ and then subjected to pulsed light activation for $16 \mathrm{~h}$. Gene expression level was normalized to the housekeeping gene, rpl13. Horizontal bars represent mean and $95 \%$ confidence interval from 4 biological replicates (NS: Not significant, $* * P<10^{-2}, * * * P<10^{-3}$ ).

A
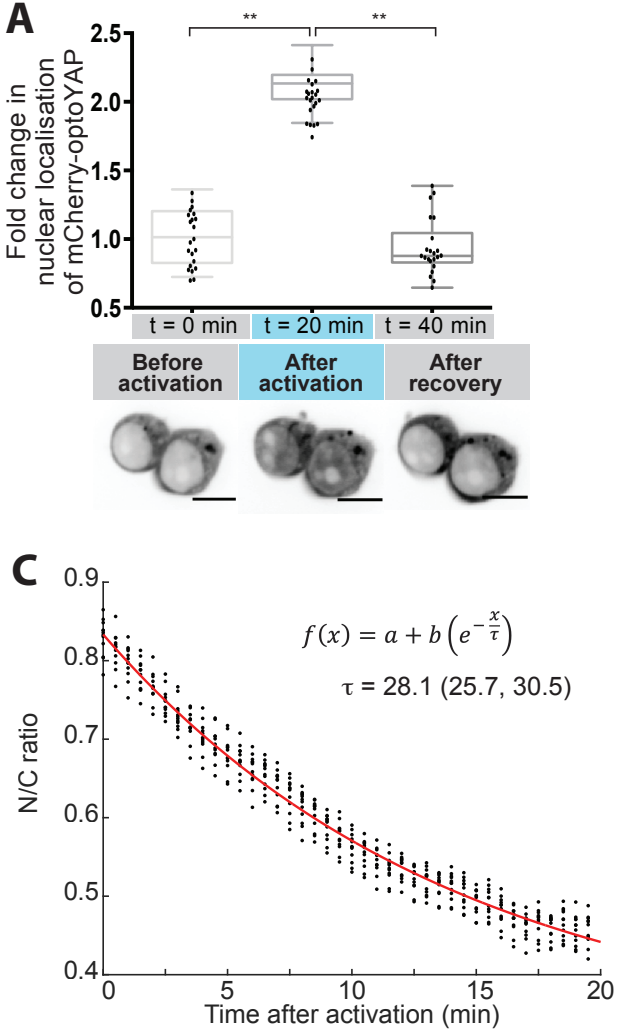

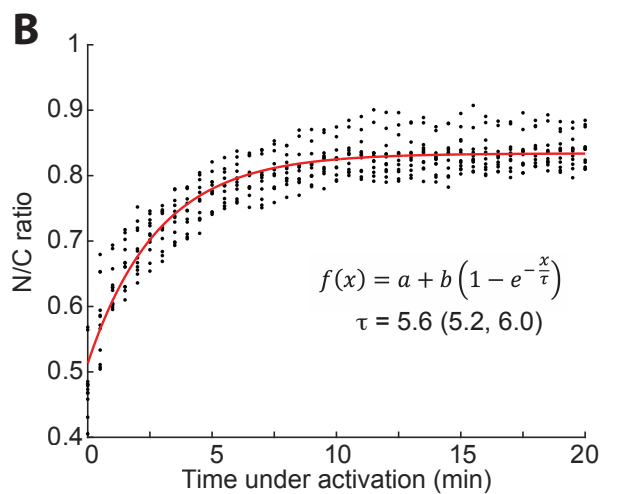

D

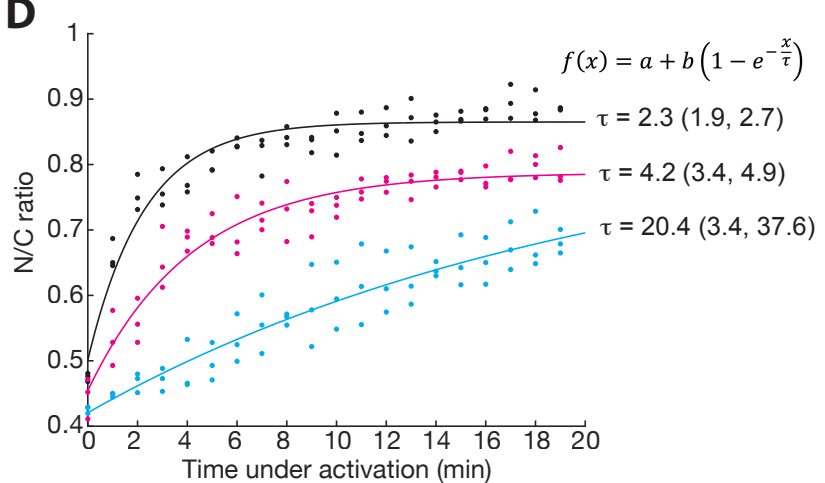

E

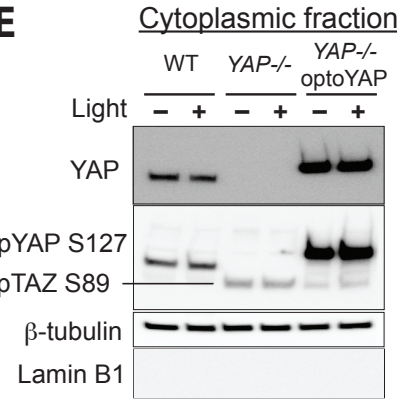

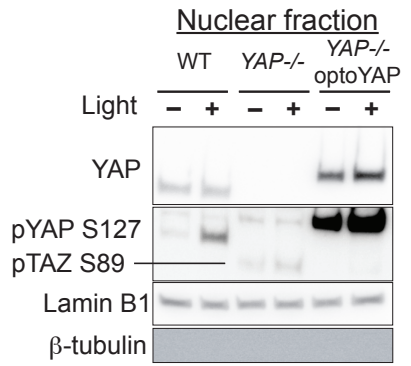

$\beta$-tubulin

Figure S1. Characterisation of optoYAP in tissue culture cells. (A) HEK293T transfected with optoYAP were subjected to activation protocol in Fig. 1A followed by recovery in the dark for $20 \mathrm{~min}$. Fold-change in nuclear localisation of mCherry-optoYAP ( $\mathrm{n}=22$ cells from 2 independent experiments). Box plots represent median and $25^{\text {th }}$ to $75^{\text {th }}$ percentiles. Bars show 
318 minimum and maximum points, ${ }^{* *} P<10^{-2}$. (B-C) Curve fitting for Fig. 1E. Red line represents

319 the exponential curve fitted to the data. Numbers in brackets represent the $95 \%$ confidence

320 interval of $\tau$. (D) Curve fitting for Fig. 1F for three different laser powers. Red line represents

321 the exponential curve fitted to the data. Numbers in brackets represent the $95 \%$ confidence

322 interval of $\tau$. (E) Western blots of MKN28 cells. MKN28 WT, $Y A P^{-/}$, and $Y A P^{-/}$cells

323 transfected with optoYAP were subjected to pulsed light activation for $48 \mathrm{~h}$. Whole cell lysate

324 from the three cell lines were separated into nuclear and cytoplasmic fractions, then probed for

325 YAP, pYAP (S127), lamin B1 and $\beta$-tubulin.

A

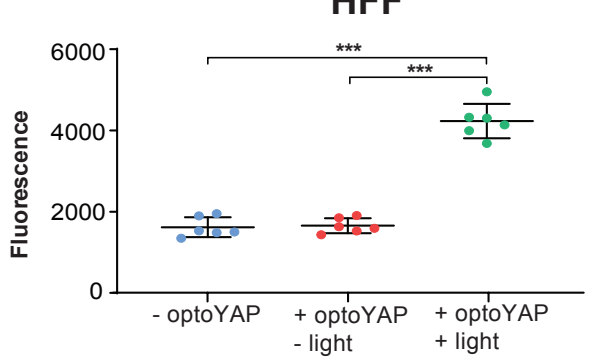

B

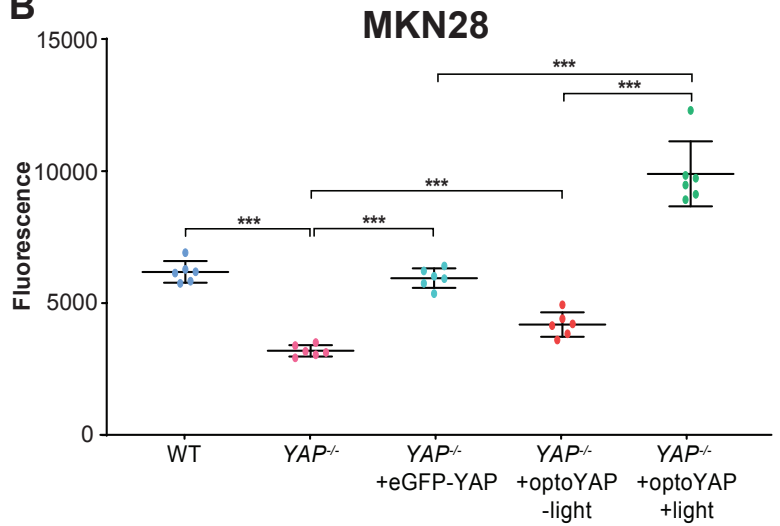

C

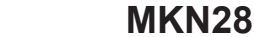

\section{MKN28}

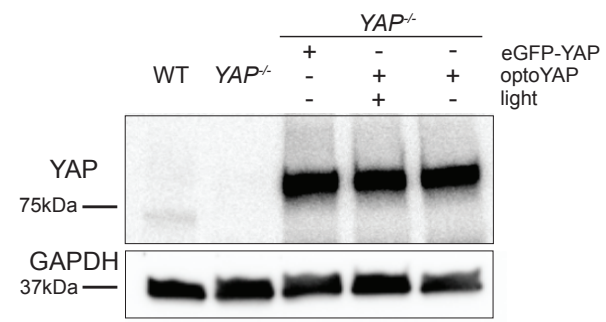
are transfected into the $Y A P^{-/}$background.

\begin{tabular}{|c|c|c|c|}
\cline { 2 - 4 } \multicolumn{1}{c|}{} & - optoYAP & $\begin{array}{l}\text { + optoYAP } \\
\text { - light }\end{array}$ & $\begin{array}{l}\text { + optoYAP } \\
\text { + light }\end{array}$ \\
\hline $\begin{array}{c}\text { Avg number } \\
\text { of colonies }\end{array}$ & 1 & 1.7 & 8.3 \\
\hline $\begin{array}{c}\text { Avg diameter } \\
\pm \text { SD (um) }\end{array}$ & $\begin{array}{c}86 \\
\pm 14.4\end{array}$ & $\begin{array}{c}86 \\
\pm 18\end{array}$ & $\begin{array}{c}193.8 \\
\pm 62.5\end{array}$ \\
\hline
\end{tabular}

\begin{tabular}{|c|c|c|c|c|c|}
\cline { 2 - 6 } \multicolumn{1}{c|}{} & WT & YAP & $\begin{array}{c}\text { YAP } \\
\text { +eGFP-YAP }\end{array}$ & $\begin{array}{c}\text { YAP- } \\
\text { +optoYAP } \\
\text { - light }\end{array}$ & $\begin{array}{c}\text { YAP- } \\
\text { +optoYAP } \\
\text { +light }\end{array}$ \\
\hline $\begin{array}{c}\text { Avg number } \\
\text { of colonies }\end{array}$ & 7.7 & 2.3 & 8.3 & 3.7 & 18.7 \\
\hline $\begin{array}{c}\text { Avg diameter } \\
\pm \text { SD (um) }\end{array}$ & $\begin{array}{c}275.2 \\
\pm 81.1\end{array}$ & $\begin{array}{c}192.9 \\
\pm 48.7\end{array}$ & $\begin{array}{c}300.4 \\
\pm 94.4\end{array}$ & $\begin{array}{c}297.1 \\
\pm 113.5\end{array}$ & $\begin{array}{c}286.9 \\
\pm 80.9\end{array}$ \\
\hline
\end{tabular}

Figure S2. Colony formation assay. (A-B) Quantification of DNA-binding fluorescent dye in HFF (A) and MKN28 (B) cells grown on soft agar. The average number and diameter of colonies formed (representative images in Fig. 3C-D) are shown in the table on the right. Error bars are s.d., $\mathrm{n}=6$ biological replicates from 2 independent experiments for each condition, $* * * P<10^{-3}$. (C) Western blots of different MKN28 cell lines. eGFP-YAP and optoYAP lines 

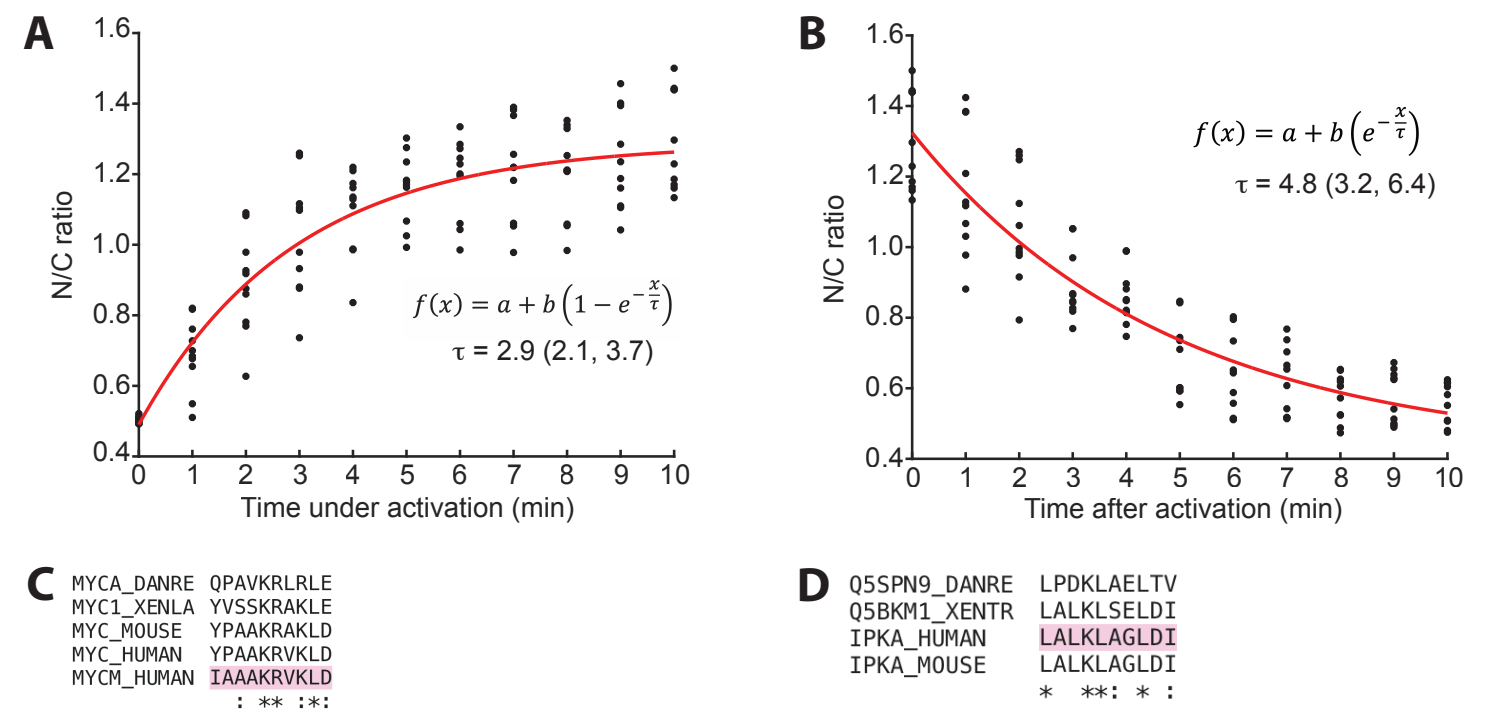

334 Figure S3. Characterisation of optofYAP in zebrafish embryos. (A-B) Curve fitting for Fig. 4C. Red line represents the exponential curve fitted to the data. Numbers in brackets represent the $95 \%$ confidence interval of $\tau$. (C-D) Multiple sequence alignments for the NLS of c-Myc (C) and NES of PKI (D) between human, mouse, frog, zebrafish and the optogenetic construct backbone (highlighted in pink).

Supplementary Video 1. HEK293T cells transfected with optoYAP under $561 \mathrm{~nm}$ only (left) or 488 and $561 \mathrm{~nm}$ (right) activation.

Supplementary Video 2. Z-stack of zebrafish embryo expressing mCherry-optofYap (magenta) and eGFP-Yap (green) before (left) and after (right) activation protocol.

\section{$\underline{\text { Methods }}$}

347 Plasmid construction

348 Optogenetic plasmid ( $\mathrm{pDN} 34$, previously reported in ${ }^{27}$ ) containing the LOV2-Ja domain was provided by Olivier Destaing. Human YAP1-1 $\delta$ was PCR amplified from pcDNA3.1 hYAP1$1 \delta$ with primers AH05 and AH06. Phusion High Fidelity DNA polymerase (ThermoScientific) was used for PCR amplification. PCR product and pDN34 were cleaved with KpnI and PmeI FastDigest restriction enzymes (ThermoScientific) for cloning. 
355

356

357

358

359

360

361

362

363

364

365

366

367

368

369

370

371

372

373

374

375

376

377

378

379

380

381

382

383

384

385

386

387

HEK293T and HFF cells were kept in phenol red-free Dulbecco's modified Eagle medium (DMEM) (Gibco) supplemented with 10\% (V/V) heat inactivated fetal bovine serum (FBS) (Hyclone) and 1\% (V/V) penicillin/streptomycin (Nacalai Tesque). MKN28 cells were kept in RPMI 1640 supplemented with 10\% (V/V) heat inactivated FBS (Hyclone) and 1\% (V/V) penicillin/streptomycin (Nacalai Tesque). All cells were maintained in $37^{\circ} \mathrm{C}$ incubator with $5 \%$ $\mathrm{CO}_{2}$.

HEK293T and HFF cells were transfected with Lipofectamine 2000 (Invitrogen) according to the manufacturer's instructions. MKN28 cells were transfected using K2 transfection system (Biontex).

\section{$\underline{\text { Zebrafish strains }}$}

All zebrafish strains were maintained according to standard fish husbandry procedures. The $A B$ wild-type strain was used in this study. All experiments with zebrafish were approved by the A*STAR Biological Resource Centre according to the Singapore National Advisory Committee on Laboratory Animal Research.

\section{$\underline{\text { Zebrafish microinjection }}$}

optofYap was cloned into a pCS2 vector backbone by restriction enzyme cloning with EcoRI and PspXI (ThermoScientific) from optoYAP. The plasmid was linearised using NotI (ThermoScientific) and capped mRNA was synthesised using mMessage Machine SP6 kit (Ambion). Embryos from AB zebrafish were collected at one-cell stage and injected with 10 pg optofYAP mRNA.

\section{Fluorescence microscopy}

Cells were visualised under the microscope about $24 \mathrm{~h}$ post transfection. Live imaging was performed on cells seeded on Iwaki glass-bottom dish and imaged on Perkin Elmer spinning disk with a LUCPlanFLN 40x/0.6 NA air objective. Injected zebrafish embryos were collected about $6 \mathrm{~h}$ post fertilisation at shield stage, dechorionated and embedded in 1\% low melting agarose dissolved in egg media. Embryos were mounted on a MatTek dish and imaged on Nikon Ti-E with Yokogawa W1 spinning disk with a PLAN APO VC 60X/1.40 NA oil immersion objective. 
388

389

390

391

392

393

394

395

396

397

398

399

400

401

402

403

404

405

406

407

408

409

410

411

412

413

414

415

416

417

418

419

420

The Nuclear/cytoplasmic ratio was measured using Fiji by drawing a region of interest (ROI) around the cell as well as its nucleus. A macro plugin was used to mask the nucleus from the cytoplasm to calculate the mean intensity in the nucleus and cytoplasm based on the ROI drawn. Nucleus was demarcated by region without mCherry signal in cells at $\mathrm{t}=0 \mathrm{~min}$ and region with highest level of mCherry in zebrafish embryos at $t=20 \mathrm{~min}$. Laser power at the focal point was measured using a power meter (Thorlabs).

\section{Light activation}

Pulsed light activation is as follows: cells are illuminated with a $1 \mathrm{~s}$ pulse of $488 \mathrm{~nm}$ laser light, followed by a $30 \mathrm{~s}$ dark phase. This pulsation is continued over $20 \mathrm{~min}$ on the Perkin Elmer or Nikon W1. For long term cell proliferation (7 d), soft agar assay (7 d) and qPCR experiments (48 h for tissue culture cells, $16 \mathrm{~h}$ for zebrafish embryos), cells were kept in cell culture incubators fitted with a LED light strip that pulsed at the same frequency.

\section{$\underline{\text { RT-qPCR }}$}

Total RNA was isolated from mammalian cell culture after $48 \mathrm{~h}$ of pulsatile light activation using RNeasy Mini Kit (Qiagen) according to the manufacturer's instructions. Complementary DNA (cDNA) was synthesised from isolated RNA using SuperScript IV Reverse Transcriptase (ThermoScientific). Zebrafish embryos injected with optofYAP at the one cell stage were subjected to pulsatile light activation and collected after $48 \mathrm{~h}$.

For zebrafish, 5 embryos were pooled into one biological replicate and a total of 4 biological replicates were obtained for each sample. RNA was extracted using TRIzol (Ambion) and purified with Direct-zol RNA kit (Zymogen). cDNA was synthesised using High Capacity RNA-to-cDNA kit (ThermoScientific).

Real-time PCR detection for both cell culture samples and zebrafish embryos were done using SYBR Green PCR Master Mix for 40 cycles in a Bio-Rad CFX96 thermal cycler. The threshold cycle $(\mathrm{Ct})$ value for each gene was normalised to the $\mathrm{Ct}$ value of a housekeeping gene, EIF1B and $r p l 13$ for cell culture and zebrafish respectively. The relative fold changes were calculated using $\Delta \Delta \mathrm{Ct}$ method. The primer sequences for target genes are listed in Table S1.

\section{$\underline{\text { Soft agar assay }}$}


421 Soft agar assay was performed using CytoSelect 96-Well Cell Transformation Assay (Cell

422 Biolabs) according to the manufacturer's instructions. The final $0.4 \%$ agar layer on which cells

423 were grown in corresponds to a Young's modulus of $<2 \mathrm{kPa}^{41} 7$ days after seeding, cell colony

424 formation was examined with EVOS Cell Imaging System (ThermoScientific) under 10X

425 magnification. Cell proliferation was measured with CyQuant NF Cell Proliferation Assay Kit

426 (ThermoScientific) according to manufacturer's instructions.

427

$428 \quad$ Cell proliferation assay

429 Cells were plated in 96-well plates at 1,000 cells per well and fluorescent intensity was 430 measured every $24 \mathrm{~h}$ post seeding for 7 days according to CyQuant NF Cell Proliferation Assay 431 (ThermoScientific) for adherent cells.

432

433 Western blotting

434 Cells were seeded in 6-well plates at 300,000 cells per well and cell lysate was harvested $48 \mathrm{~h}$ 435 post seeding. Nuclear and cytoplasmic fractions were separated using NE-PER Nuclear and 436 Cytoplasmic Extraction Reagents (ThermoScientific) according to manufacturer's instructions 437 for adherent cells. Protein concentration of both fractions were measured using Pierce BCA 438 Protein Assay Kit (ThermoScientific). 15 ug of protein were separated by 4-20\% Mini Protean 439 TGX SDS-polyacrylamide gels (Bio-Rad) at $120 \mathrm{~V}$ for $1 \mathrm{~h}$ and transferred onto $0.2 \mathrm{um}$ PVDF 440 membranes (Bio-Rad) at $90 \mathrm{~V}$ for $1 \mathrm{~h}$. Membranes were blocked with $3 \%(\mathrm{~W} / \mathrm{V})$ BSA in TBST 441 for $1 \mathrm{~h}$ at room temperature (RT) and incubated overnight at $4^{\circ} \mathrm{C}$ with primary antibodies in $3 \%$ $442(\mathrm{~W} / \mathrm{V})$ BSA in TBST. Membranes were washed 3 times for 5 min with TBST and incubated 443 with either anti-mouse or rabbit HRP-conjugated secondary antibodies in 3\% (W/V) BSA in 444 TBST for $1 \mathrm{~h}$ at RT. Membranes were washed 3 times for 5 min with TBST after secondary 445 antibody incubation and chemiluminescence signal was detected using Clarity Western ECL 446 Substrate (Bio-Rad). All relevant information on antibodies used in this study are listed in 447 Table S2.

449 Curve fitting

450 Time constant $(\tau)$ of optoYAP import and export from the nucleus was fitted using the curve 451 fitting function fit in MATLAB. Nuclear import rate $\left(\tau_{\mathrm{a}}\right)$ was fitted with $f(x)=a+$ $b\left(1-e^{-\frac{x}{\tau}}\right)$ and export rate $\left(\tau_{\mathrm{r}}\right)$ was fitted with $f(x)=a+b\left(e^{-\frac{x}{\tau}}\right)$. 
Supplementary Methods

455 Table S1. List of primers used.

\begin{tabular}{|c|c|c|}
\hline Name & Sequence & Use \\
\hline $\begin{array}{l}\text { AH05 YAP N-term } \\
\text { fwd KpnI }\end{array}$ & $\begin{array}{l}\text { GTAGCAGGTACCGGAGGGGGGG } \\
\text { GGGCCCGGAGGCGGGGGGAGGC } \\
\text { GGGGGATCCCGCCACCATGGAT } \\
\text { CCCGGGCAGCAG }\end{array}$ & Cloning YAP into pDN34 \\
\hline $\begin{array}{l}\text { AH06 YAP N-term } \\
\text { rev PmeI }\end{array}$ & $\begin{array}{l}\text { ACGTAGGTTTAAACCTATAACC } \\
\text { ATGTAAGAAAGCTTTCTTTATCT } \\
\text { AGCTTGGTG }\end{array}$ & Cloning YAP into pDN34 \\
\hline EIF1B_L & TGATGCAACTAAGGGTGACG & RT-qPCR for cell culture \\
\hline EIF1B_R & CAGTGTCTTTCTGCCGTTCC & RT-qPCR for cell culture \\
\hline ANKRD1_L & ATGCTTGGAAGGACATTTGG & RT-qPCR for cell culture \\
\hline ANKRD1_R & TGGCTGTGGATTCAAGCATA & RT-qPCR for cell culture \\
\hline CTGF_L & TGCATCCGTACTCCCAAAAT & RT-qPCR for cell culture \\
\hline CTGF_R & ATGTCTTCATGCTGGTGCAG & RT-qPCR for cell culture \\
\hline CYR61_L & TCCCTGTTTTTGGAATGGAG & RT-qPCR for cell culture \\
\hline CYR61_R & GAGCACTGGGACCATGAAGT & RT-qPCR for cell culture \\
\hline PT49 vgll41 fwd & GTGCGTTGAAGATTCAGGAATG & $\begin{array}{l}\text { RT-qPCR for zebrafish } \\
\text { embryos }\end{array}$ \\
\hline PT50 vgll41 rev & CACTCTGTCGCTGGTCATGTGC & $\begin{array}{l}\text { RT-qPCR for zebrafish } \\
\text { embryos }\end{array}$ \\
\hline PT51 cyr61 fwd & ACGCGAAGATGTTTGCTTGG & $\begin{array}{l}\text { RT-qPCR for zebrafish } \\
\text { embryos }\end{array}$ \\
\hline PT52 cyr61 rev & CCGGCCCTCTGATTTAGCTC & $\begin{array}{l}\text { RT-qPCR for zebrafish } \\
\text { embryos }\end{array}$ \\
\hline PT53 ctgfa fwd & $\begin{array}{l}\text { GTGTTCACCTGGTGTAAGCCTAG } \\
\text { TTC }\end{array}$ & $\begin{array}{l}\text { RT-qPCR for zebrafish } \\
\text { embryos }\end{array}$ \\
\hline PT54 ctgfa rev & $\begin{array}{l}\text { ACCGTCCAGACACGTGCACTGG } \\
\text { TAT }\end{array}$ & $\begin{array}{l}\text { RT-qPCR for zebrafish } \\
\text { embryos }\end{array}$ \\
\hline PT55 rpl13 fwd & TAAGGACGGAGTGAACAACCA & $\begin{array}{l}\text { RT-qPCR for zebrafish } \\
\text { embryos }\end{array}$ \\
\hline
\end{tabular}




\begin{tabular}{|l|l|l|}
\hline PT56 rpl13 rev & CTTACGTCTGCGGATCTTTCTG & $\begin{array}{l}\text { RT-qPCR for zebrafish } \\
\text { embryos }\end{array}$ \\
\hline PT57 vgll2b fwd & GACAAAGAGGTCGGAGAGGC & $\begin{array}{l}\text { RT-qPCR for zebrafish } \\
\text { embryos }\end{array}$ \\
\hline PT58 vgll2b rev & CGCCGACTGCTTGAGGATAA & $\begin{array}{l}\text { RT-qPCR for zebrafish } \\
\text { embryos }\end{array}$ \\
\hline PT59 arhgap27 fwd & CACAATCTCTGCAGCCAGGA & $\begin{array}{l}\text { RT-qPCR for zebrafish } \\
\text { embryos }\end{array}$ \\
\hline PT60 arhgap27 rev & CCGCCAGATTTCCACTGACT & $\begin{array}{l}\text { RT-qPCR for zebrafish } \\
\text { embryos }\end{array}$ \\
\hline
\end{tabular}

Table S2. List of antibodies used.

\begin{tabular}{|l|l|l|l|}
\hline Target protein & Supplier & Catalogue number & Dilution \\
\hline YAP (D8H1X) & Cell signalling technology & $\# 14074$ & $1: 1000$ \\
\hline pYAP (S127) & Cell signalling technology & $\# 4911$ & $1: 1000$ \\
\hline GAPDH & Cell signalling technology & $\# 5174$ & $1: 5000$ \\
\hline$\beta$-tubulin & Cell signalling technology & $\# 2128$ & $1: 1000$ \\
\hline Lamin B1 & Abcam & ab16048 & $1: 5000$ \\
\hline Anti-rabbit IgG & Cell signalling technology & $\# 7074$ & $1: 5000$ \\
\hline Anti-mouse IgG & Cell signalling technology & $\# 7076$ & $1: 5000$ \\
\hline
\end{tabular}

\section{$\underline{\text { References }}$}

461 1. Pan, D. Hippo signaling in organ size control. Genes Dev. 21, 886-897 (2007).

462 2. Harvey, K. F., Pfleger, C. M. \& Hariharan, I. K. The Drosophila Mst ortholog, hippo, restricts growth and cell proliferation and promotes apoptosis. Cell 114, 457-467 (2003). coordinately regulates cell proliferation and apoptosis by inactivating Yorkie, the Drosophila homolog of YAP. Cell 122, 421-434 (2005).

468 4. Dong, J. et al. Elucidation of a universal size-control mechanism in Drosophila and mammals. Cell 130, 1120-1133 (2007).

470 5. Hilman, D. \& Gat, U. The evolutionary history of YAP and the Hippo/YAP pathway. 
Mol. Biol. Evol. 28, 2403-2417 (2011).

6. Chan, E. H. Y. et al. The Ste20-like kinase Mst2 activates the human large tumor suppressor kinase LATS1. Oncogene 24, 2076-2086 (2005).

7. Callus, B. A., Verhagen, A. M. \& Vaux, D. L. Association of mammalian sterile twenty kinases, Mst1 and Mst2, with hSalvador via C-terminal coiled-coil domains, leads to its stabilization and phosphorylation. FEBS J. 273, 4264-4276 (2006).

8. Hergovich, A., Schmitz, D. \& Hemmings, B. A. The human tumour suppressor LATS1 is activated by human MOB1 at the membrane. Biochem. Biophys. Res. Commun. 345, 50-58 (2006).

9. Basu, S., Totty, N. F., Irwin, M. S., Sudol, M. \& Downward, J. Akt phosphorylates the Yes-Associated Protein, YAP, to induce interaction with 14-3-3 and attenuation of p73-mediated apoptosis. Mol. Cell 11, 11-23 (2003).

10. Kanai, F. et al. TAZ: a novel transcriptional co-activator regulated by interactions with 14-3-3 and PDZ domain proteins. EMBO J. 19, 6778-6791 (2000).

11. Zhao, B., Li, L., Tumaneng, K., Wang, C. Y. \& Guan, K. L. A coordinated phosphorylation by Lats and CK1 regulates YAP stability through SCF $\beta$-TRCP. Genes Dev. 24, 72-85 (2010).

12. Totaro, A., Panciera, T. \& Piccolo, S. YAP/TAZ upstream signals and downstream responses. Nat. Cell Biol. 20, 888-899 (2018).

13. Fu, V., Plouffe, S. W. \& Guan, K. L. The Hippo pathway in organ development, homeostasis, and regeneration. Curr. Opin. Cell Biol. 49, 99-107 (2017).

14. Heallen, T. R., Kadow, Z. A., Kim, J. H., Wang, J. \& Martin, J. F. Stimulating cardiogenesis as a treatment for heart failure. Circ. Res. 124, 1647-1657 (2019).

15. Xin, M. et al. Hippo pathway effector Yap promotes cardiac regeneration. Proc. Natl. Acad. Sci. 110, 13839-13844 (2013).

16. Wu, S., Huang, J., Dong, J. \& Pan, D. hippo encodes a Ste-20 family protein kinase that restricts cell proliferation and promotes apoptosis in conjunction with salvador and warts. Cell 114, 445-456 (2003).

17. Morikawa, Y., Heallen, T., Leach, J., Xiao, Y. \& Martin, J. F. Dystrophin-glycoprotein complex sequesters Yap to inhibit cardiomyocyte proliferation. Nature 547, 227-231 (2017).

18. Zanconato, F., Cordenonsi, M. \& Piccolo, S. YAP/TAZ at the roots of cancer. Cancer Cell 29, 783-803 (2016).

19. Yizhar, O., Fenno, L. E., Davidson, T. J., Mogri, M. \& Deisseroth, K. Optogenetics in 
neural systems. Neuron 71, 9-34 (2011).

20. Tischer, D. \& Weiner, O. D. Illunimating cell signaling with optogenetic tools. Nat. Rev. Mol. Cell Biol. 15, 551-558 (2014).

21. Toettcher, J. E., Weiner, O. D. \& Lim, W. A. Using optogenetics to interrogate the dynamic control of signal transmission by the Ras/Erk module. Cell 155, 1422-1434 (2013).

22. Katsura, Y. et al. An optogenetic system for interrogating the temporal dynamics of Akt. Sci. Rep. 5, 1-10 (2015).

23. Viswanathan, R. et al. Optogenetic inhibition of Delta reveals digital Notch signalling output during tissue differentiation. EMBO Rep. 20, 1-13 (2019).

24. Huang, A., Amourda, C., Zhang, S., Tolwinski, N. S. \& Saunders, T. E. Decoding temporal interpretation of the morphogen Bicoid in the early Drosophila embryo. Elife 6, 1-21 (2017).

25. Kerjouan, A. et al. Control of SRC molecular dynamics encodes distinct cytoskeletal responses by specifying signaling pathway usage. J. Cell Sci. 134, 1-19 (2021).

26. Niopek, D., Wehler, P., Roensch, J., Eils, R. \& Di Ventura, B. Optogenetic control of nuclear protein export. Nat. Commun. 7, 1-9 (2016).

27. Niopek, D. et al. Engineering light-inducible nuclear localization signals for precise spatiotemporal control of protein dynamics in living cells. Nat. Commun. 5, 1-11 (2014).

28. Zhao, B. et al. Inactivation of YAP oncoprotein by the Hippo pathway is involved in cell contact inhibition and tissue growth control. Genes Dev. 21, 2747-2761 (2007).

29. Zhao, B. et al. TEAD mediates YAP-dependent gene induction and growth control. Genes Dev. 22, 1962-1971 (2008).

30. Wang, H. B., Dembo, M. \& Wang, Y. L. Substrate flexibility regulates growth and apoptosis of normal but not transformed cells. Am. J. Physiol. Cell Physiol. 279, C1345-C1350 (2000).

31. Yang, B. et al. Stopping transformed cancer cell growth by rigidity sensing. Nat. Mater. 19, 239-250 (2020).

32. Qiao, Y. et al. YAP regulates actin dynamics through ARHGAP29 and promotes metastasis. Cell Rep. 19, 1495-1502 (2017).

33. Kimelman, D., Smith, N. L., Lai, J. K. H. \& Stainier, D. Y. R. Regulation of posterior body and epidermal morphogenesis in zebrafish by localized Yap1 and Wwtr1. Elife 6, 1-29 (2017). 
539

540

541

542

543

544

545

546

547

548

549

550

551

552

553

554

555

556

557

34. Dowbaj, A. M. et al. An optogenetic method for interrogating YAP1 and TAZ nuclearcytoplasmic shuttling. bioRxiv (2020). doi:doi.org/10.1101/2020.06.08.140228

35. Uda, Y. et al. Efficient synthesis of phycocyanobilin in mammalian cells for optogenetic control of cell signaling. Proc. Natl. Acad. Sci. 114, 11962-11967 (2017).

36. Moya, I. M. \& Halder, G. Hippo-YAP/TAZ signalling in organ regeneration and regenerative medicine. Nat. Rev. Mol. Cell Biol. 20, 211-226 (2019).

37. Johnson, R. \& Halder, G. The two faces of Hippo: targeting the Hippo pathway for

38. Grijalva, J. L. et al. Dynamic alterations in Hippo signaling pathway and YAP regenerative medicine and cancer treatment. Nat. Rev. Drug Discov. 13, 63-79 (2014). activation during liver regeneration. Am. J. Physiol. Liver Physiol. 307, G196-G204 (2014).

39. Aharonov, A. et al. ERBB2 drives YAP activation and EMT-like processes during cardiac regeneration. Nat. Cell Biol. 22, 1346-1356 (2020).

40. Monroe, T. O. et al. YAP partially reprograms chromatin accessibility to directly induce adult cardiogenesis in vivo. Dev. Cell 48, 765-779 (2019).

41. Li, C., Huang, Z. \& Wang, R. K. Elastic properties of soft tissue-mimicking phantoms assessed by combined use of laser ultrasonics and low coherence interferometry. Opt. Express 19, 10153-10163 (2011). 